\title{
Practice Forum
}

\section{Reducing hospital acquired Clostridium difficile associated diarrhoea in an acute care facility}

\author{
Dan Costello \\ Nanaimo Regional General Hospital, Vancouver Island, British Columbia, Canada
}

Int J Infect Contr 2007, 3:1 doi:10.3396/03-01-07-007 Available from: http://www.ijic.info

\begin{abstract}
:
Hospital acquired Clostridium difficile associated diarrhoea (CDAD) was determined to be a longstanding historical concern at Nanaimo Regional General Hospital, with an infection rate nearly double the Canadian national average for similarly sized facilities. During 2004/2005, a significant outbreak of CDAD fuelled an urgent response.
\end{abstract}

The facilities, Infection Prevention \& Control (IP\&C) program deficiencies, and organizational culture issues were considered as strategies for improvement were contemplated. The multifaceted programmatic approach and partnerships with key stakeholders and patrons were instrumental in successfully reducing nosocomial CDAD and sustaining this low rate. The timeline for this initiative was 18 months beginning in July, 2005, with evaluation completed in December, 2006.

\section{Introduction}

Nanaimo Regional General Hospital (NRGH) is a 243bed acute care facility in the city of Nanaimo on the east coast of Vancouver Island, British Columbia, Canada. The hospital serves a local population of just over 100,000 in addition to acting as a referral centre to about 200,000 residents of rural communities.

The nursing tower of the hospital was constructed in the 1960s. There are only 16 private rooms with dedicated toilets, with the balance being two- and four-bed rooms. The ratio of patients to toilets on the nursing wards is about 3:1.

As a result of the dated facilities, equipment and supplies have outstripped storage capacity, often spilling into hallways; many surfaces are damaged and not easily cleaned (Figure 1); and over census patients are commonly nursed in the halls behind portable curtains without running water or dedicated toilet facilities.

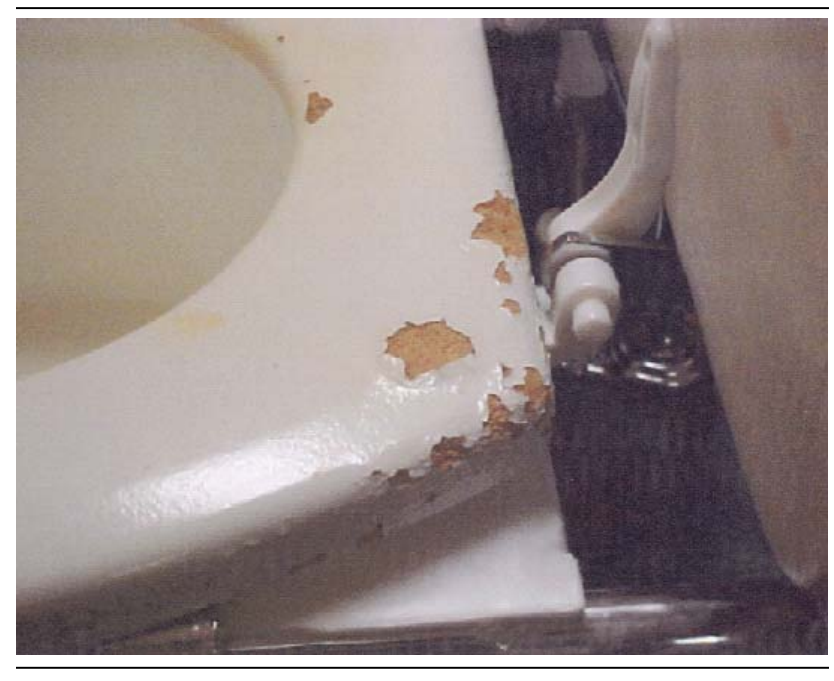

Figure 1: Damaged surfaces impede adequate cleaning 
Based on records from the previous 6 years, the rate of nosocomial CDAD at NRGH was as high as 10.2 cases/1000 admissions per year, nearly double the Canadian national average of 5.9 cases/1000 admissions for facilities of similar size and service delivery.

In 2004/2005 the hospital experienced a significant outbreak of CDAD in a medical unit, with an attack rate of $70 \%$. In addition there were three other clusters on three separate medical and surgical units. These outbreaks captured the attention of the local media and the public began to express concerns about the safety of the hospital.

\section{Background}

There was little evidence of a structured Infection Prevention \& Control (IP\&C) program at NRGH prior to 2003. Human resources for IP\&C consisted of one parttime employee who had other work duties and very little surveillance data could be found for any infections.

IP\&C reference resources for nursing and other staff was outdated. Protective barrier wear was limited to gowns and gloves found perched outside private rooms, on chairs, or handrails. Commode chairs were rusty with worn through seats. No assigned responsibility or procedures for cleaning and disinfecting of commodes were evident. There was little understanding of the need to use infection control precautions for diarrhoea illness.

There was a widely held view that patients acquired CDAD in hospital as a consequence of illness and antibiotic therapy. Further, many nurses felt there was little that could be done to prevent this from occurring. Staff were aware of environmental influences, such as worn out commodes, that contribute to the risk of infection transmission but verbalized a sense of powerlessness in their ability to effect change.

Despite this, once the concerning CDAD rates were brought to their attention, staff were receptive to the need to reduce the incidence of infection and were supportive of recommendations for solutions to the problem.

\section{Methodology}

A Participatory Action Research (PAR) model was used to guide the development and implementation of a remedial action plan. The model is described by Loiselle et $\mathrm{al}^{1}$ as a research methodology designed to empower participants through the process of problem solving. It is circular in nature with questions designed to find solutions followed by implementation.

The first step in evaluation was to return to the participant with questions related to effectiveness and acceptance of the solution. Our team used this principled approach whenever the opportunity presented. The seeds of change were planted for some, whilst others were empowered by the knowledge that they were co-authors of the solution.

\section{Actions}

- Historic rates for nosocomial CDAD obtained from laboratory archives served to benchmark the high rate of infection. Collaboration with the laboratory helped refine a definition for nosocomial CDAD and assured sensitivity of the data findings and ongoing surveillance.

\section{Education}

Following focus group discussions with nurses on a CDAD outbreak ward, a self-directed learning module was developed and distributed to all nursing staff. Key points included:

o the concerning incidence of infection; the impact of illness on morbidity and risk for mortality;

o a working definition of CDAD to assure early identification and timely implementation of control measures;

o modes of transmission of the organism; enhanced housekeeping measures; procedures for commode cleaning and the importance of hand washing with soap and water;

o most importantly, the importance of better communication between nurses. At the suggestion of staff, a special "infection control" space was developed on the nursing Kardex.

- A patron medical supply company supported the education campaign with cash prizes awarded for nursing units with best completion of learning modules. Feedback was given to all staff on their participation (Figure 2). Clinical leaders provided further encouragement to participate in this challenge to keep pace with others.

- Using feedback obtained from patients and families during an experience on an outbreak unit, a third year nursing student developed an information brochure to better meet their information needs.

- The strategic plan was summarized on a poster board by an undergraduate nursing student who led further discussions on all nursing units.

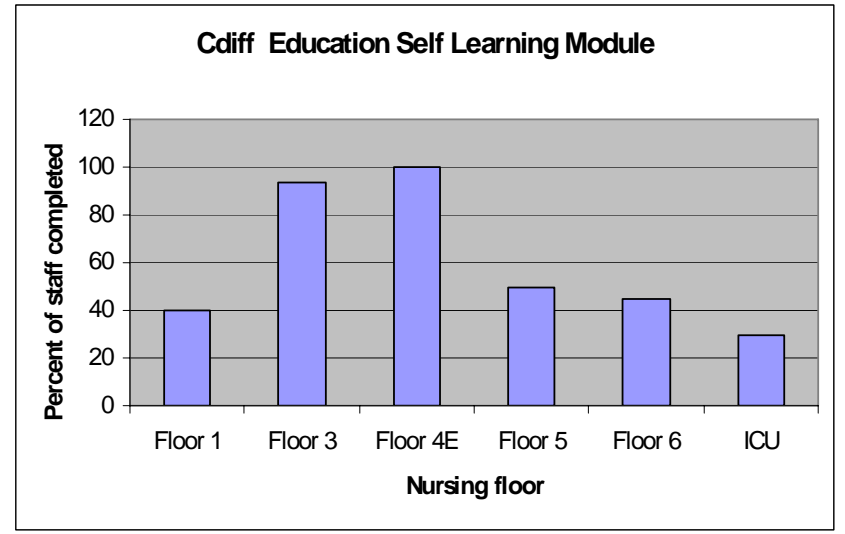

Figure 2: Participation of staff in self-learning module 


\section{Equipment}

- In partnership with a medical equipment manufacturer, a new commode chair was constructed. Design features to facilitate ease of cleaning and automated washing include: a stainless steel frame with sealed or welded joints; sealed castors; and polypropylene mesh webbing for the seat back. A bracket was mounted on the rear frame to hold disinfectant wipes. All commode chairs in the facility were replaced with this model and additional chairs were purchased to assure one could be dedicated to each patient in need. A focus group of nurses, physical and occupational therapists were asked to evaluate the model for functional design.

- Infection control barrier supply carts were purchased (Figure 3). Impervious gowns were added for wet, heavy work, and supplies, signs and reference materials were organized to facilitate ease of use.

- Washer disinfectors were purchased to replace flush hoppers, which would eliminate manual cleaning. Their use would also assure disinfection of commode basins and bedpans .

- An automated washer was installed for terminal cleaning of commodes. Responsibility for this work was assumed by housekeeping services.

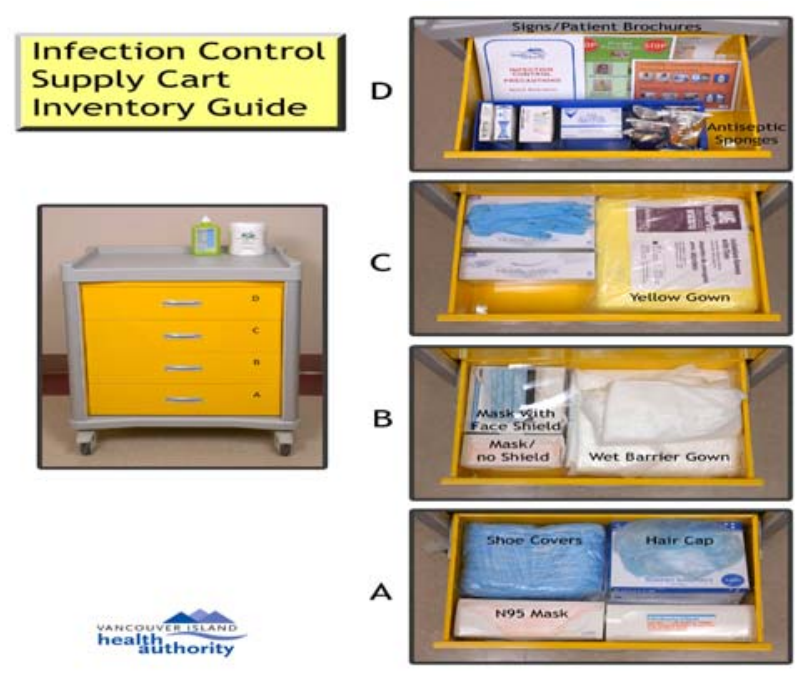

Figure 3: Infection control barrier supply carts

\section{Enhanced cleaning}

Collaboration with the newly assigned housekeeping contractor resulted in a change of cleaning practices. A two-step cleaning/disinfection of all patient room surfaces, using first accelerated hydrogen peroxide then bleach, was adopted.

\section{Antibiotic Stewardship}

- Hospital antibiotic utilization was reviewed through the Pharmacy and Therapeutics Committee. A goal of $20 \%$ reduction in use of broad-spectrum antibiot- ics, in particular, fluoroquinolones, over two years was established. An audit is in progress and a report on this is expected in the spring of 2007.

- The Hospital Epidemiologist presented to the Medical Advisory Committee on the strategic plan and followed this with a communication to area physicians.

\section{Discussion}

Early in 2006, the hospital faced an additional challenge as the Health Region underwent restructuring. The local Board of Directors for NRGH and surrounding rural hospitals was dissolved and decision making was centralized. As an indirect consequence, the IP\&C team lost their ability to easily influence capital purchases and building improvements at the local level. This served to delay full implementation of our strategic plan.

Despite the use of the PAR model, there were a number of staff who verbalized disapproval of the commode chair design features and replacement of open hoppers with automation. Much time and energy was expended on addressing these objections and supporting rationale for the improvements.

The IP\&C team chose an ambitious plan to remedy high rates of nosocomial CDAD transmission. It did so with the slimmest of human resources. One Infection Control Practitioner, two nursing students part-time and parttime clerical support.

Absences and departures of Infection Control Practitioners from other sites within the newly restructured Health Region created gaps in time dedicated to NRGH as the IP\&C practitioner at NRGH was asked to support these sites for urgent needs. This further impeded our ability to implement and evaluate the program in person. Thankfully, the clinical leaders on the units shouldered the burden of this responsibility.

\section{Conclusion}

Within eight weeks of implementing the education campaign and housekeeping changes, a measurable reduction of CDAD transmission was realized. Rates of nosocomial CDAD were further reduced once the additional measures were in place. This low rate is sustained through the latter half of 2006 (Figure 4).

The strategic plan and outcomes have been shared with Infection Control Practitioners across the Province of British Columbia through the Provincial Infection Control Network (PicNet) and the education module is posted on the PicNet website.

The impact of this program has supported a paradigm shift in thinking about infections in relation to their risk of transmission to patients. The medical and nursing admission assessment now includes a formal review of infection symptoms. Where infection control precautions were once implemented on the basis of positive 
microbiology reports, staff now implement precautions upon identification of symptoms. The impact of this second program has also influenced a significant downward trend of nosocomial MRSA transmission (Figure 5) despite an almost exponential rise in laboratory documented community-associated MRSA infections presenting to the Emergency Room and being admitted to the hospital.

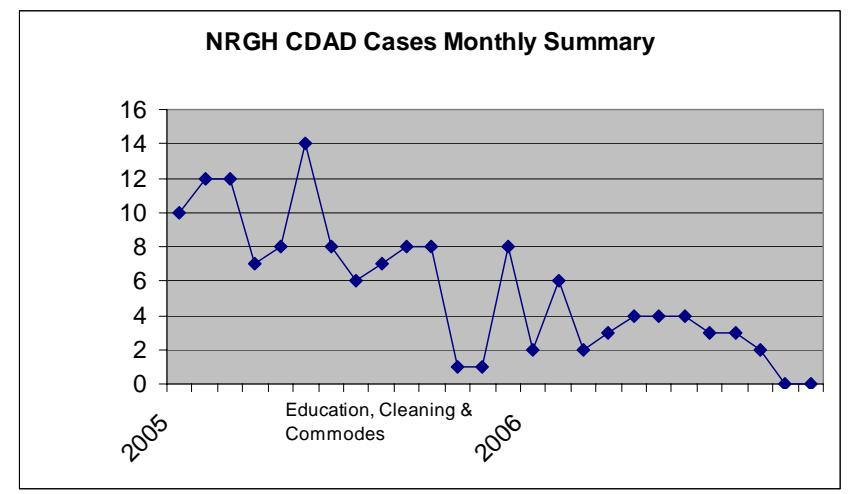

Figure 4: CDAD Cases 2005-6

Nosocomial transmission of CDAD was creating much public anxiety about the safety of NRGH. The added morbidity in patients was estimated to be causing in the range of 750 additional days of hospital stay per year. This reality was straining the public trust. The IP\&C team faced many challenges in bringing this vicious cycle to an end, including an aging infrastructure, organizational culture, limited IP\&C team resources and regional health care reform.

Despite these realities, the results have been encouraging. A paradigm shift in thinking about and acting on infectious symptoms has created behavioural change in all practitioners. All healthcare workers have worked together to accomplish this goal and, in this sense, are the larger IP\&C team. It is hoped that these measures will

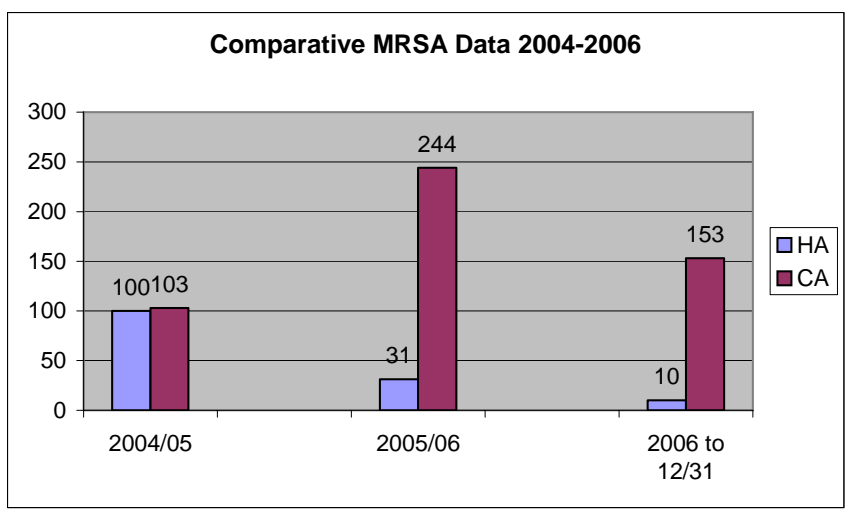

Figure 5: Methicillin-resistant S. aureus cases

HA - hospital acquired

CA - community acquired help to rebuild the public trust in NRGH as a safe place in which to receive healthcare.

\section{References}

1. Loiselle, C.G., Profetto-McGrath, J., Polit, D.F., Beck, C.T. (2006) Canadian Essentials of Nursing Research (2 ${ }^{\text {nd }}$ Ed.), Lippincott Williams \& Wilkins: Philadelphia.

This publication has been adapted from the original submission by the authors for the Oxoid Infection Control Team of the Year Awards 2006/2007 (www.oxoid.com) and is being reproduced with permission of Oxoid Ltd. 